\title{
Manifestation of Diabetes Mellitus Type 1: A Questionnaire to Evaluate the Acceptance of Initial Intravenous Therapy
}

\author{
Katrin Steul*, Laurenz Corvers, Joachim Pohlenz, Dorothee Maria Kieninger-Baum \\ Children University Hospital, Mainz, Germany \\ Email: ${ }^{*}$ steul@uni-mainz.de
}

Received 25 August 2014; revised 20 September 2014; accepted 17 October 2014

Academic Editor: Thomas Stief, Institute of Laboratory Medicine and Pathobiochemistry, Germany

Copyright (C) 2014 by authors and Scientific Research Publishing Inc.

This work is licensed under the Creative Commons Attribution International License (CC BY).

http://creativecommons.org/licenses/by/4.0/

(c) (i) Open Access

\section{Abstract}

For families suddenly confronted with the diagnosis of a lifelong chronic disease, implementing a continuous iv. infusion represents restriction of movement and possibly also a psychological burden. We designed a questionnaire to evaluate the perception of pediatric patients and their parents for this treatment as part of a diabetes manifestation. Patients and their parents treated in the diabetes outpatient clinic at children's university hospital in Mainz were asked for their opinion about "iv. infusion" and "frequency of blood taking" during their first stay in the hospital upon manifestation of diabetes. They assigned scores from 1 to 10 for the following parameters: "delivery of diagnosis", "communication by doctor", "execution of training", "atmosphere during diabetes training", "frequency of blood taking", "iv. infusion" and "start of sc. insulin application". Parents gave relatively high ratings. Parameters such as "frequency of blood taking" were rated with 6.1 on average. Ultimately, "frequency of blood taking", "delivery of diagnosis" (6.11) and "iv. infusion of insulin" (6.5) scored lower than for instance "atmosphere of diabetes training" (7.95). Children awarded scores of 3.72 for "iv. infusion", on average, whereas they scored $\mathbf{7 . 1 5}$ for the "doctor's communication" and 7.28 for "diabetes training". Asked if a decision in favor of a subcutaneous insulin injection right at the beginning of the therapy would have been preferable, parents were undecided. Therefore it cannot be concluded that an instant subcutaneous therapy has psychological advantages or disadvantages. The patients themselves (aged 12 - 17 years) were undecided, too, $(58.3 \%)$ when asked for their preference of the subcutaneous insulin regime right at the beginning of the therapy. Nevertheless, the continuous infusion of insulin iv. was rated poorly by them. When planning further studies on this topic, the following questions should be analyzed: If the initial therapy had been started subcutaneously, would the manifestation be assessed differently by the family? Is the patients' poor rating of the iv. insulin injection reproduci-

*Corresponding author.

How to cite this paper: Steul, K., Corvers, L., Pohlenz, J. and Kieninger-Baum, D.M. (2014) Manifestation of Diabetes Mellitus Type 1: A Questionnaire to Evaluate the Acceptance of Initial Intravenous Therapy. Journal of Diabetes Mellitus, 4, 350-358. http://dx.doi.org/10.4236/jdm.2014.44048 
ble? Do other factors more greatly influence the decision for or against iv. insulin injection at the start of the therapy, such as the time needed for caretaking for the iv. injection by the nurses or possible side effects of an iv. injection? How can the start of the therapy be organized, when starting with sc. therapy, in particular regarding the first communication with patient and family?

\section{Keywords}

\section{Diabetes Mellitus Type 1, Manifestation, iv. Insulin Therapy}

\section{Introduction}

Onset of Diabetes mellitus type 1 occurs in a multitude of cases in childhood or during adolescence. Guidelines exist for the event that the manifestation of diabetes is combined with a diabetic ketoacidosis [1]-[3]. These guidelines comprise a rehydration-process, substitution of electrolytes, insulin management and monitoring. If the diabetes manifestation takes place without a ketoacidosis, there is no distinct guideline process starting therapy. Commencement of therapy via subcutaneous insulin application is one possibility. Furthermore, the ISPAD Consensus Guidelines (2009) recommend the use of intravenous insulin application only for ketoacidotic states or in case of surgical procedures, not for the standard situation of diabetes manifestation.

The existing guidelines (ISPAD, BSPED, DDG) do not provide guidance on the start of the therapy via subcutaneous insulin; nor do they provide guidance regarding the preferable method of insulin application: intravenous vs. subcutaneous.

The German diabetes association's guideline (DDG, Deutsche Diabetes Gesellschaft) "Diagnostics, Therapy and Monitoring of Diabetes mellitus during childhood and adolescence (Diagnostik, Therapie und Verlaufskontrolle des Diabetes mellitus im Kindes- und Jugendalter) only includes a short paragraph about the start of therapy: Insulin therapy should be started immediately... and a diabetes team with an expertise in paediatric diabetology should be involved as quickly as possible [4]. There is no position for or against immediate subcutaneous insulin application.

The British Society for Paediatric Endocrinology and Diabetes (BSPED) circulates Guidelines for the Management of Diabetic Ketoacidosis (2009). These include comprise emergency management, clinical assessment, management of fluids and electrolytes and the management of complications such as cerebral edema. With regard to the application of insulin, it recommends a continuous low-dose intravenous infusion with 0.05 or 0.1 units/kg/hour [1] [2]. An equivalent guideline for the start of the therapy without diabetic ketoacidosis does not exist. This guideline gives a detailed and helpful overview of DKA management. It is in line with the latest ISPAD (International Society for Pediatric and Adolescent Diabetes) guidelines from 2009.

The ISPAD Clinical Practice Consensus Guidelines (2009) focus on the "Insulin treatment in children and adolescents with diabetes" [3]. This guideline gives information about the different insulins which exist, mode and duration of action, storage and injection sites. In the section about iv. infusion, it is postulated that iv insulin is only used in crisis situations such as diabetic ketoacidosis and during surgical procedures. No detailed procedure for the start of therapy after manifestation with subcutaneous insulin is defined.

The ISPAD Guideline "Diabetic ketoacidosis in children and adolescents with diabetes" (2009) provides a definition, assessment and management information [5]. In the section about insulin therapy it is stated that in circumstances where a continuous iv. administration is not possible, an hourly or 2-hourly sc. or im. administration is possible (safe and effective). An initial dose of 0.3 units $/ \mathrm{kg}$ insulin lispro or aspart followed by $0.1 \mathrm{units} / \mathrm{kg}$ every hour or $0.15-0.2$ units/kg every 2 hours is recommended. But this only applies to the management of diabetic ketoacidosis. This statement does not cover the case of commencing therapy without DKA.

In the course of revising the internal guidelines for diabetic patients at the University of Mainz children's hospital, we reconsidered the in-house scheme for commencing therapy. Until then, the therapy began by conducting an iv. insulin application in a standard situation, even if no sign of diabetic ketoacidosis was present. The start of sc. Insulin normally takes place (provided the patient is clinically in a stable state, no signs of ketoacidosis etc.) after a family has had contact with one of the doctors from the diabetes team and has had enough time to discuss their questions about the diabetes therapy. One of the reasons for this is that starting of sc. insulin appli- 
cation before a detailed conversation between the family and the diabetes team has taken place, has often proved unsatisfying for the families.

Nevertheless, the application of an iv. catheter might be a an additional (psychological) burden for the patients. In the absence of investigations into that issue, we aimed to analyze the patient's (and their parent's) perception of various factors relating to diabetes manifestation, including iv. infusion.

This family questionnaire complements and extends another analysis which was published in 2012 [6]. In this analysis we asked German and Austrian centres with expertise in pediatric diabetology about their procedure for starting therapy in diabetes manifestation situations. They were asked about their decision-making process in terms of favouring or rejecting iv. insulin infusion at the start of therapy.

Our ambition is to gain information to finally develop an appropriate scheme for therapy commencement in diabetes manifestation (without diabetic ketoacidosis).

\section{Methods}

In this explorative investigation, patients and their parents were questioned about their experience of diabetes manifestation. All of them were using the diabetes outpatient clinic of the University of Mainz children's hospital at the time of questioning. They were interviewed using standardized questionnaires between.

The patients involved were at least 8 years old at this point in time. They were separated into two groups according to their age and provided with different questionnaires (8 - 11 years, 12 - 17 years). The patients aged 18 y. or more were interviewed using a questionnaire with the same questions as the 12 - 17 years olds'. All patients and their parents were asked for their informed consent/assent. The investigation was approved by the local ethics committee. Patients younger than 8 years of age were not questioned, but their parents were asked to participate. In total, 156 persons took part. This included 48 patients and 108 parents. 17 patients were in the age group 8 - 11 (10 girls, 7 boys). 23 adolescents were in the age group 12 - 17 (12 female, 11 male). 8 participating patients were older than 18 ( 4 female, 4 male). The parents' group consisted of 59 women and 49 men coming from 61 families (including 12 single mothers, 2 single fathers).

Data on the patients' gender, duration of diabetes, age and age upon diabetes manifestation and therapy (ICT, CSII etc.) were evaluated. The patients aged 12 and above and their parents were then asked for their opinions in the following categories.

In the first category, they were asked what they could remember about their first stay in the hospital: First contact with diabetes team (first information about diabetes), Doctor who made the diagnosis, Diabetes team involved in the diabetes training, Contents of diabetes training, Frequency of blood taking in the first hours/days, Infusion of iv. insulin, Transition to sc. therapy.

Patients were also asked if they remembered being instructed not to be allowed to eat.

In a second category, they were asked to assign scores from 1 to 10 ( 1 for a negative experience and 10 for a good one).

They could assign scores for Delivery of the diagnosis, Communication by the doctor, Execution of diabetes training, Atmosphere during training, Frequency of blood taking in the first hours/days, Infusion of iv. insulin, Transition to sc. insulin.

In a third category, the parents were asked whether, in their opinion, the application of sc. insulin right at the beginning of the therapy would have been preferable to an iv. insulin infusion. In the fourth category they were asked if they think it would have been preferable for their children.

The adult patients (>17 years) and the adolescent patients (aged 12 - 17) were only asked in a third category regarding a possible preference for sc. insulin application at the beginning.

In addition to the general information about gender, diabetes duration and age, the 8 - 11 year olds were asked for feedback in three categories.

First they were asked if they could remember their first stay in hospital. In the second category, they were asked which details of their first stay they could remember: The iv. insulin infusion, Transition to sc. application, Frequency of blood taking, Not being allowed to eat in the first few hours, Diabetes training.

And in a third category they were asked which of the above mentioned aspects had caused them particular frustration.

For analysis and statistics, we used the software SPSS, version 19 by IBM. 


\section{Results}

In the first category it we asked which details the parents could remember. Generally, the mothers remembered more than the fathers. For example 84.7 percent of the mothers remember the names of the persons (nurses, doctors) who conducted the training versus 73.5 percent of the fathers (Figure 1). Details regarding the start of therapy (iv. insulin infusion (57.4\%) and transition to sc. therapy) (58.3\%) were generally not as well remembered as the other items: first contact with diabetes team (75\%), doctor who made diagnosis (66.7\%), diabetes team involved in the training (nurses, doctors) (79.6\%), contents of diabetes training (77.8\%), frequency of blood taking (79.6\%).

In the group of patients aged 12 - 17, the differences between boys and girls were more distinct. For example, $75 \%$ of the girls could remember the name of the doctor who made the diagnosis versus $27.3 \%$ of the boys (Figure 2). The initial contact with the diabetes team and the first information could generally not be remembered that well (30.4\% versus $75 \%$ in the parents' group).

The trend that the items of the therapy start (iv. insulin infusion and transition to sc. therapy) are not as well remembered as the other items could be seen in the parents' group. In the group of 12 - 17 year olds-even if the 18 years and older are added—-that trend is not evident (Figure 3).

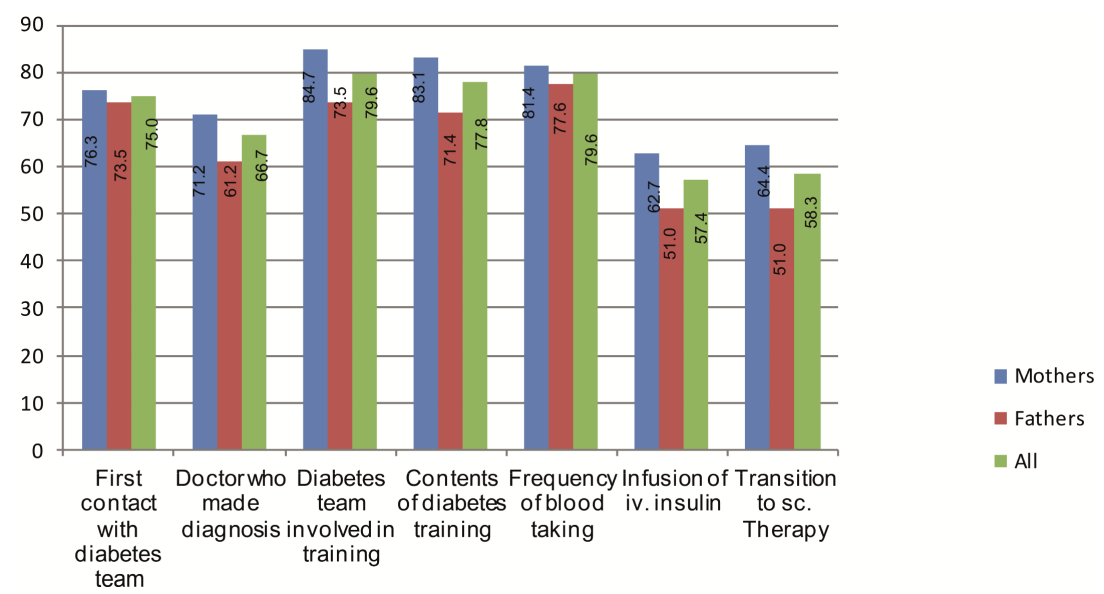

Figure 1. Percentage of parents remembering a specific detail of their first stay in hospital.

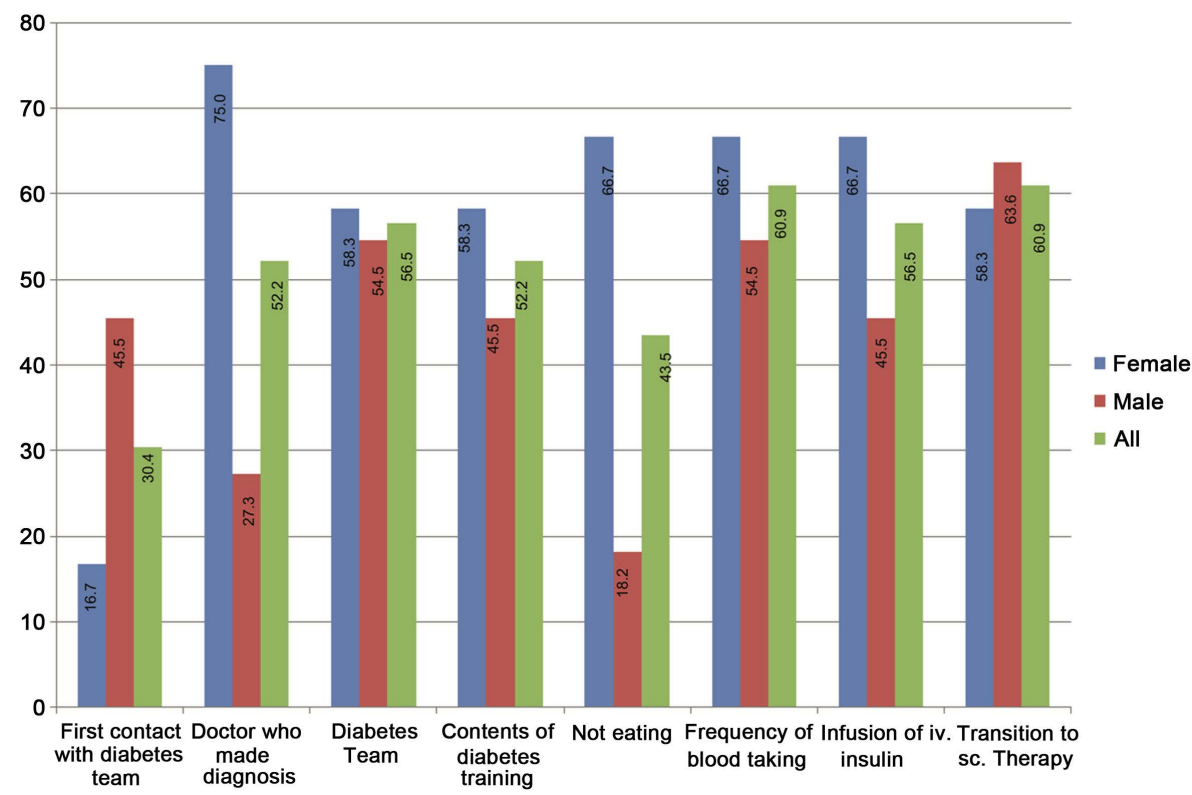

Figure 2. Percentage of patients (aged 12 - 17 years) remembering a specific detail of their first stay in hospital. 


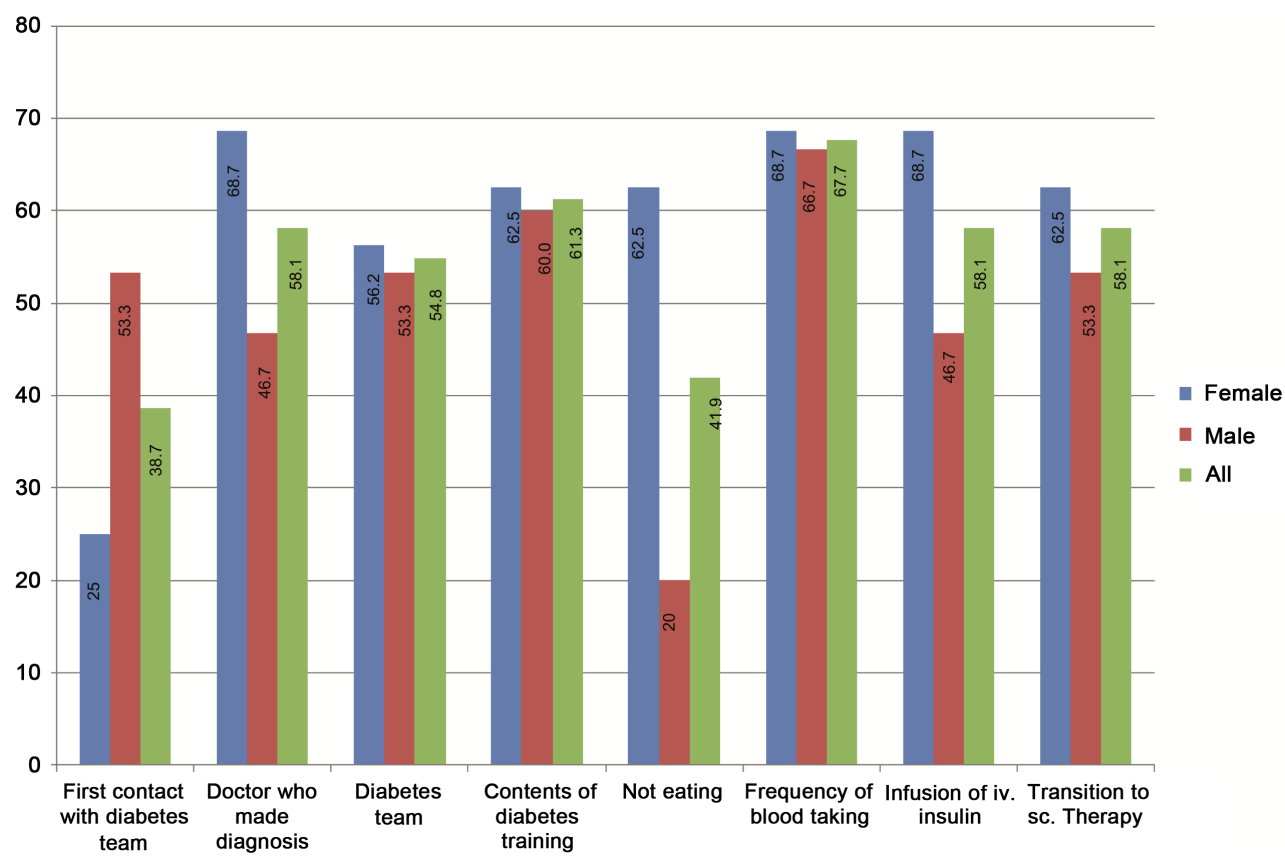

Figure 3. Percentage of patients (aged 12 - 17 years and older than 17) remembering a specific detail of their first stay in hospital.

In a second category of questions, parents and patients 12 and above were asked to award scores from 1 to 10 for certain aspects of their first stay in hospital. The parents gave ranging scores between 6 and 8. It resulted in no clear differentiation between the items. For example, items which might have, in our opinion, easily achieved high scores, such as the atmosphere during training (mean value 7.95) scored little more than the iv. insulin infusion (mean value 6.5) analysed in this questionnaire. Items which, in our opinion, were more connected with negative memories for the families, such as the frequency of blood taking, still scored 6.1 points. Since the score in this examination was defined as 1 for a bad experience and 10 for a good one, this result seems puzzling (Table 1).

Interestingly, in the group of patients aged 12 - 17 there was a recognizable differentiation between some items. They got, for example, a mean score of 7.28 for execution of training (Table 2). The infusion of iv. insulin, the item which was evaluated in this analysis, only scored 3.19. This score is clearly lower than, for example, those for atmosphere during training (6.81) and a communication by doctor (7.15). If the point scoring is defined as 1 for a bad experience and 10 for a good one, scores higher than 5 represent a broadly good experience. From this point of view, also in the patients group, it seems puzzling that frequency of blood taking scored more than 5 (mean value 5.38).

This characteristic is also evident if you add the adult patients (18 years and older) (Table 3): Infusion of iv. insulin scored lower than the others (3.62). Frequency of blood taking was awarded a value higher than 5 (5.17).

Patients aged between 8 and 11 were asked which details of their first stay they could remember and which aspects they found highly unpleasant (Figure 4 and Figure 5). Only 5.9 percent of patients in this age group rated the diabetes training as very unpleasant (Figure 5). Frequency of blood taking and not being allowed to eat was rated as very unpleasant by $64.7 \%$ and $52.9 \%$ of patients respectively in this age group. iv. infusion of insulin and transition to sc. therapy were both remembered as rather unpleasant in $35.3 \%$ of cases. Ultimately, no preference for a therapy option (subcutaneously or intravenously) can be identified in this age group.

In a third category, parents and patients aged 12 years and above were asked whether it would have been preferable if the therapy had been started with the application of sc. insulin. In this category, the parents as well as the patients themselves seemed undecided. $32.4 \%$ of the parents answered the question with "undecided". $13 \%$ did not even answer the question. Other responses were equally divided. $27.8 \%$ voted with "no", $26.9 \%$ answered with "yes".

The patients (aged 12 - 17 years) were mostly "undecided” (47.8\%), even though the iv. insulin infusion was 
Table 1. Parents’ scores arithmetic mean.

\begin{tabular}{cc}
\hline Parents' scores & Arithmetic mean \\
\hline Delivery of diagnosis & 6.11 \\
Communication by the doctor & 7.48 \\
Execution of training & 7.79 \\
Atmosphere during training & 7.95 \\
Frequency of blood taking & 6.1 \\
Infusion of iv. insulin & 6.5 \\
Transition to sc. insulin & 7.23 \\
\hline
\end{tabular}

Table 2. Patients' (aged 12 - 17 years) scores as an arithmetic mean on a scale from 1 to 10 .

\begin{tabular}{cc|}
\hline Patients' scores & Arithmetic mean \\
\hline Delivery of diagnosis & 6.24 \\
Communication by the doctor & 7.15 \\
Execution of training & 7.28 \\
Atmosphere during training & 6.81 \\
Frequency of blood taking & 5.38 \\
Infusion of iv. insulin & 3.72 \\
Transition to sc. insulin & 6.55 \\
\hline
\end{tabular}

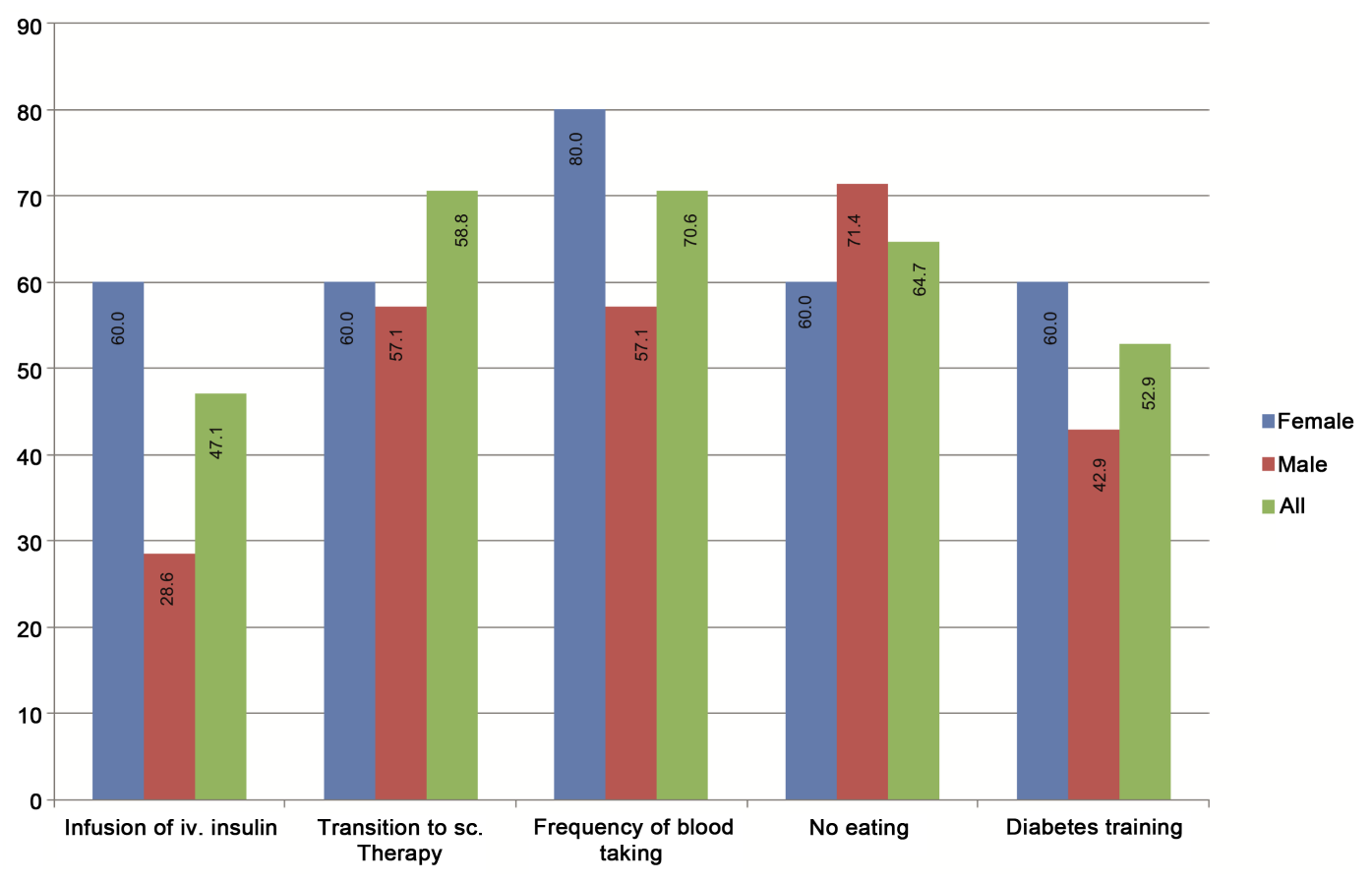

Figure 4. Percentage of patients (8 - 11 years) remembering a specific detail of their first stay in hospital. 
Table 3. Patients' (aged 12 - 17 years, older than 17 years) scores as an arithmetic mean on a scale from 1 to 10 .

\begin{tabular}{cc}
\hline Patients' scores & Arithmetic mean \\
\hline Delivery of diagnosis & 6.32 \\
Communication by the doctor & 7.43 \\
Execution of training & 7.55 \\
Atmosphere during training & 7.17 \\
Frequency of blood taking & 5.17 \\
Infusion of iv. insulin & 3.62 \\
Transition to sc. insulin & 6.19 \\
\hline
\end{tabular}

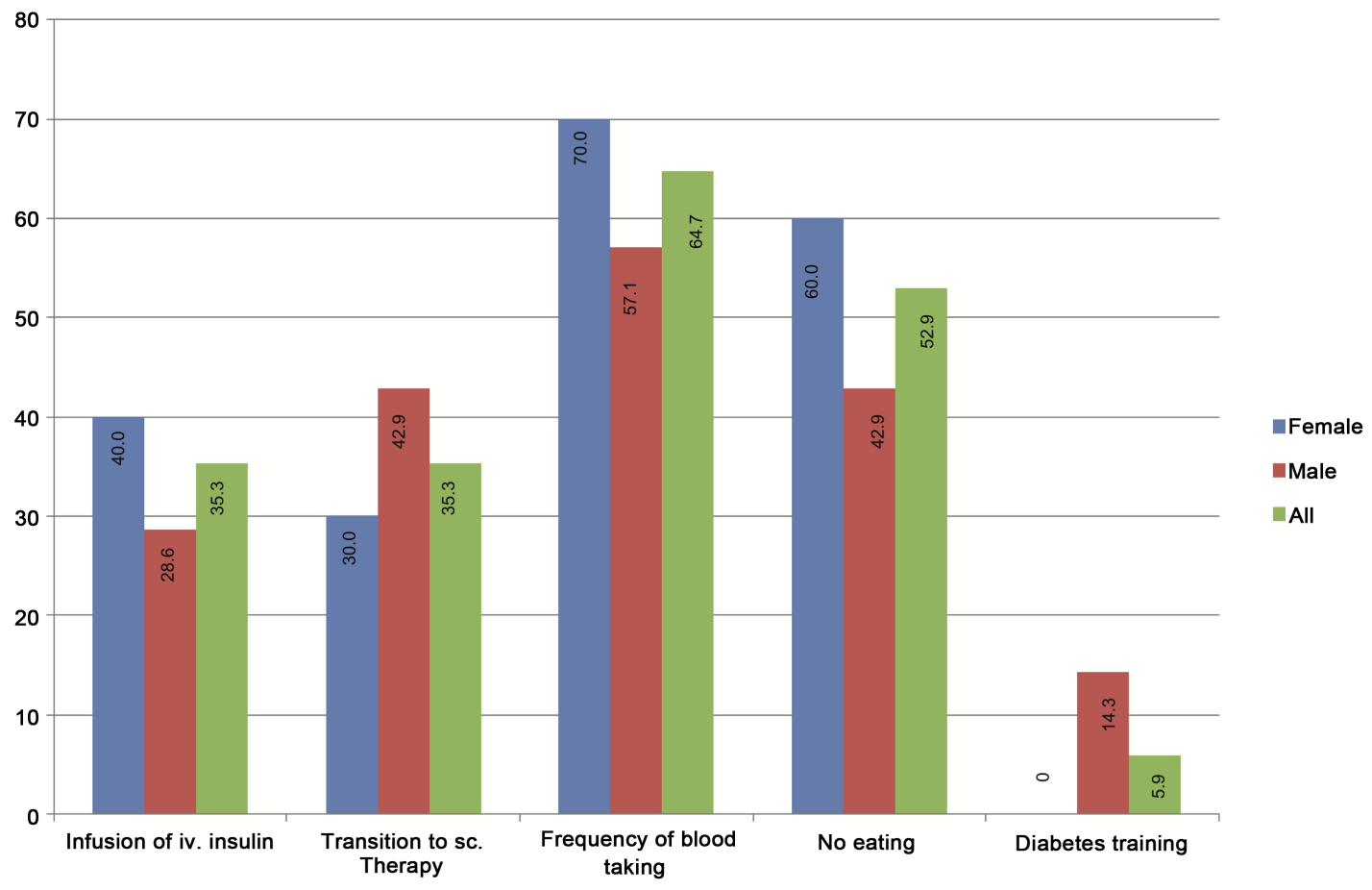

Figure 5. Percentage of patients ( 8 - 11 years) particularly frustrated by a specific aspect of their first stay in hospital.

given lower scores than in the adult group (in Category 2). Only 39.1\% had a clear opinion on this matter (21.7\% for no, $17.4 \%$ for yes).

\section{Discussion}

In the first question category, patients and parents were asked which details of their first stay in hospital they could remember. Generally, two trends could be detected in this analysis.

1. The female study subjects remembered more details than the male study subjects.

2. In the parents' group, the items constituting the start of therapy (iv. insulin infusion, transfer to sc. therapy) were not as well remembered as the other ones.

Since this is a descriptive analysis it cannot be concluded that there is a level of significance.

Some research work has been done about psychosomatic issues in the diabetic population [7] [8]. The perception of certain details of the patients' first stay in hospital has not been analyzed so far. This makes it hard to set this evaluation in context. Regarding the first question category, it may be of interest if the observed trend that the details of the start of therapy (iv. insulin infusion, transfer to sc. therapy) in the parents' group are not that 
well remembered, can be reproduced. It may be seen as an indication that the details of the therapy (iv. insulin application versus sc. application)—at least in the parents' group — did not make such a strong impact when compared to other items such as the first gaining of information. This correlates with our observation that occurrences such as the first information about diabetes given by the diabetes team normally have a very strong impact on the parents. The memory of these events might mask other details of the first stay in hospital.

In the second category, parents and patients aged 12 and above were asked for their evaluation of certain details of their first stay in hospital. In this category, they could give scores between 1 and 10. We asked for details which (in our opinion) could have achieved rather high ratings such as the atmosphere of training, as well as aspects which represent negative experiences such as the frequency of blood taking.

In this category, parents gave, in mean, scores between 6.1 and 7.95. Since items such as the frequency of blood taking adhered to a rather bad experience, it is astounding that parents gave 6.1 points in mean. Ultimately, 6.1 is a score above 5, which would stand for a neutral experience. Perhaps the parents did not want to criticize details of their first contact with the diabetes team in whose supervision they have been since then. But (again) it has to be pointed out that this analysis was a descriptive one. In a further analysis these questions should be paraphrased to avoid misunderstandings.

Surprisingly, in the patients' group aged 12 - 17, and when the adult patients were added, a differentiation could be observed. Patients were asked for the same details of their first stay: delivery of diagnosis, communication by the doctor, execution of training, atmosphere of training, frequency of blood taking, infusion of iv. insulin and transition to sc. insulin. They gave an average score of 5.38 for the frequency of blood taking representing a neutral experience. They gave rather high ratings for the communication by the doctor and the execution of training $(7.15,7.28)$. But the aspect which was actually the main focus of this analysis, i.e. the infusion of iv. insulin, was only given 3.72 points in mean. This result might indicate that the iv. insulin infusion at the start of therapy is a strong additional burden, at least for the patients themselves. This correlates with the cognition that the patients frequently ask early to end the iv. infusion during hospital stay. The reasons for this were not part of this analysis. It might be connected with the binding character of an iv. infusion (problems moving with the iv. infusion). Another explanation might be pain at the injection site or the experience of applying the iv. Smaller children might perceive the iv. as scary. Both the low scoring in this analysis, and the possible reasons, should be re-evaluated in further studies (with endpoint character).

The patients aged 8 to 11 were asked in a separate questionnaire. First they were asked which aspect of their first stay they could remember. They then were asked which aspect of their first stay had caused them particular frustration. In this category, it must be discussed whether the statements of the young patients who could not remember one of the situations in question can be used. For example, if a subject stated he/she did not remember the iv. insulin infusion (as shown in Figure 4) at all, the statement as to whether one aspect had caused particular frustration is represented in Figure 5? Disregarding this problem in the patients aged 8 - 11, a preference for a therapy option could not be seen. The iv. insulin infusion and the transition to sc. insulin were both rated as rather unpleasant in only $35.3 \%$ of cases. The frequency of blood taking was evaluated as rather unpleasant in $64.7 \%$ of cases.

In the third question category for parents and patients aged 12 and above, it was asked if the start of the therapy via sc. insulin application had been preferable. Both groups (parents and patients) and the parents, when asked if it had been preferable for their children, were undecided. This seems puzzling, especially in the group of the patients, because this group made a clear differentiation in the second question category by giving a bad score for the iv. insulin infusion. We expected a clear voting against iv. insulin infusion in this category as well. Again this needs to be re-evaluated in further studies building on our analysis. Perhaps the questions should be combined, for example:

"If you gave a low score for iv. insulin infusion ( $<5$ points), would you have preferred if the insulin had been applied sc. from the very beginning?”

This analysis aimed at an evaluation of the first stay in hospital of young type 1 diabetes patients. It was designed as a descriptive analysis to gain a first impression of the patients' and their parents' perception of essential parts of their first in-patient stay. The analysis complements another one published in 2012 [6]. In this examination, German and Austrian pediatric centers/hospitals with an expertise in pediatric diabetology were asked about their procedure for the start of therapy. The bottom line was that the majority of centers use an iv. insulin infusion during their patients' first stay in hospital, even though there is no clear evidence nor a recommendation for this approach. The current analysis shows first evidence that the iv. insulin infusion might be an 
additional burden for the young type 1 diabetics. The issues discussed above should be re-evaluated in future controlled multi-center trials.

\section{References}

[1] Dunger, D.B., Sperling, M.A., Acerini, C.L., et al. (2004) European Society for Paediatric Endocrinology/Lawson Wilkins Pediatric Endocrine Society. Consensus Statement on Diabetic Ketoacidosis in Children and Adolescents. Pediatrics, 113, e133-e140. http://dx.doi.org/10.1542/peds.113.2.e133

[2] Edge, J.A. (2009) BSPED Recommended DKA Guidelines 2009. http://www.bsped.org.uk/clinical/docs/DKAGuideline.pdf

[3] Bangstad, H.J., Danne, T., Deeb, L.C., et al. (2009) ISPAD Clinical Practice Consensus Guidelines 2009 Compendium. Insulin Treatment in Children and Adolescents with Diabetes. Pediatric Diabetes, 10, 82-99.

[4] Neu, A., Beyer, P., Bürger-Büsing, J., et al. (2010) Diagnostik, Therapie und Verlaufskontrolle des Diabetes mellitus im Kindes- und Jugendalter. Diabetologie, 5, S155-S165. http://dx.doi.org/10.1055/s-0030-1262636

[5] Wolfsdorf, J., Craig, M.E., Daneman, D., et al. (2009) Diabetic Ketoacidosis in Children and Adolescents with Diabetes. Pediatric Diabetes, 10, 118-133. http://dx.doi.org/10.1111/j.1399-5448.2009.00569.x

[6] Steul, K., Dudda, M., Pohlenz, J. and Kieninger, D. (2013) Manifestation eines Diabetes mellitus Typ 1 im Kindesund Jugendalter. Therapiebeginn in deutschen pädiatrisch-diabetologischen Zentren. Monatsschrift Kinderheilkd, 161, 21-26. http://dx.doi.org/10.1007/s00112-012-2774-6

[7] Sassmann, H., De Hair, M., Danne, T., et al. (2012) Reducing Stress and Supporting Positive Relations in Families of Young Children with Type 1 Diabetes: A Randomized Controlled Study for Evaluating the Effects of the DELFIN Parenting Program. BMC Pediatrics, 20, 152. http://dx.doi.org/10.1186/1471-2431-12-152

[8] Ashraff, S., Siddiqui, M.A. and Carline, T.E. (2013) The Psychosocial Impact of Diabetes in Adolescents: A Review. Oman Medical Journal, 28, 159-162. http://dx.doi.org/10.5001/omj.2013.46 
Scientific Research Publishing (SCIRP) is one of the largest Open Access journal publishers. It is currently publishing more than 200 open access, online, peer-reviewed journals covering a wide range of academic disciplines. SCIRP serves the worldwide academic communities and contributes to the progress and application of science with its publication.

Other selected journals from SCIRP are listed as below. Submit your manuscript to us via either submit@scirp.org or Online Submission Portal.
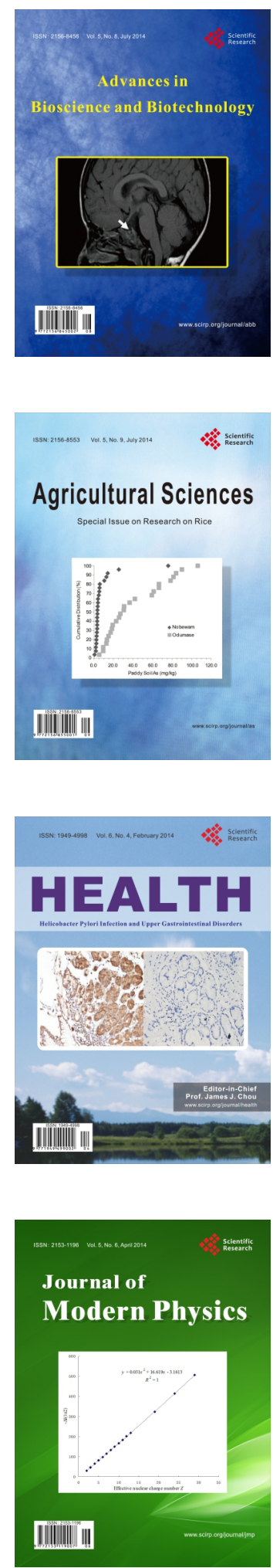
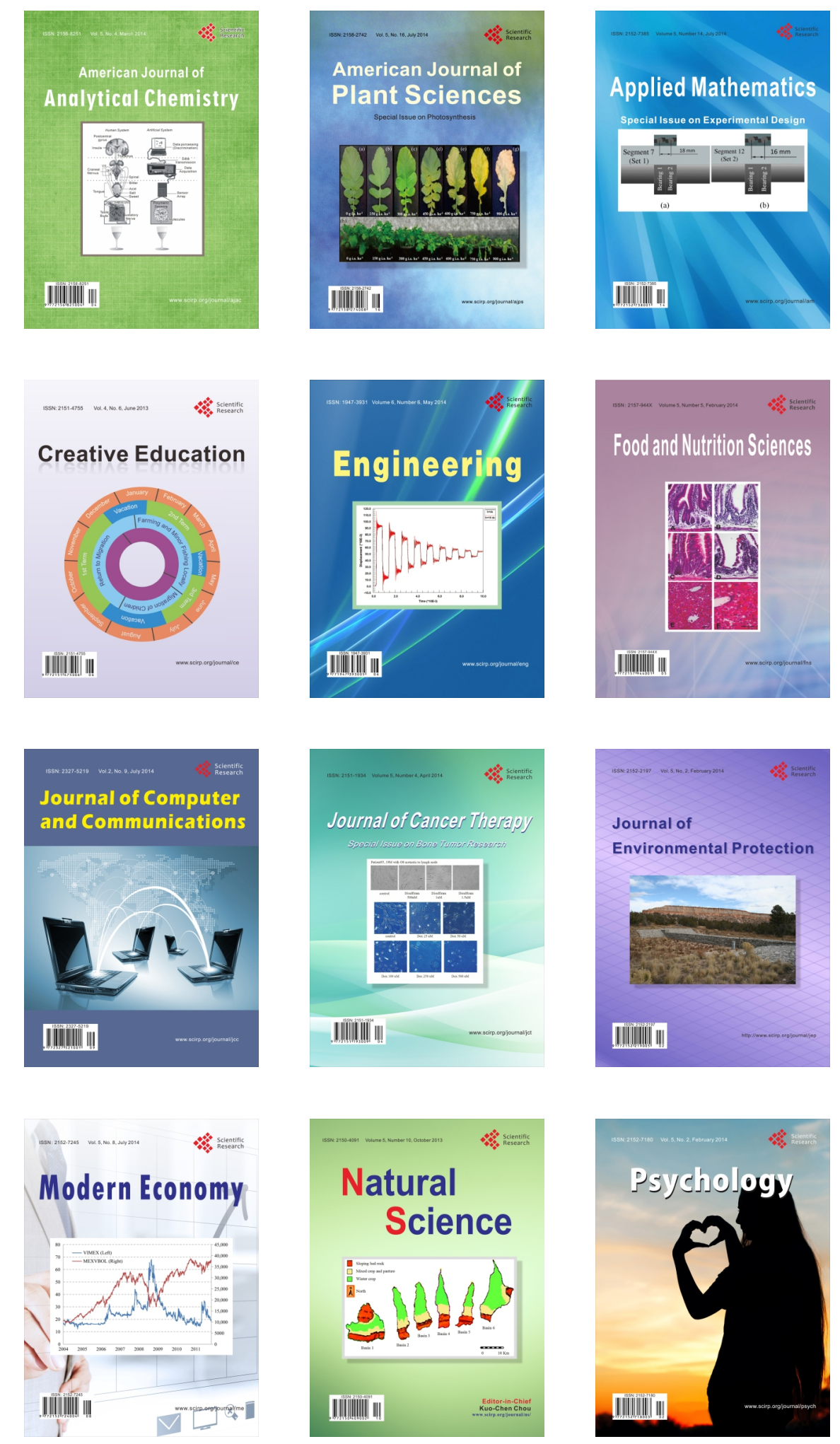